\title{
Response: Commentary: Statistical Modeling for the Prediction of Infectious Disease Dissemination With Special Reference to COVID-19 Spread
}

\author{
Subhash Kumar Yadav ${ }^{1 *}$ and Yusuf Akhter ${ }^{2 *}$ \\ ${ }^{1}$ Department of Statistics, School of Physical and Decision Sciences, Babasaheb Bhimrao Ambedkar University, Lucknow, \\ India, ${ }^{2}$ Department of Biotechnology, School of Life Sciences, Babasaheb Bhimrao Ambedkar University, Lucknow, India
}

Keywords: distribution fitting models, time series regression models, epidemiological models of disease, parameters, estimation, prediction

\section{A Commentary on}

\section{OPEN ACCESS}

Edited by:

Monica Catarina Botelho, Instituto Nacional de Saúde Doutor

Ricardo Jorge (INSA), Portugal

Reviewed by:

Subhas Khajanchi,

Presidency University, India

*Correspondence:

Subhash Kumar Yadav

drskystats@gmail.com

Yusuf Akhter

yusuf@daad-alumni.de

Specialty section:

This article was submitted to

Infectious Diseases - Surveillance,

Prevention and Treatment,

a section of the journal

Frontiers in Public Health

Received: 25 September 2021

Accepted: 30 December 2021

Published: 31 January 2022

Citation:

Yadav SK and Akhter Y (2022)

Response: Commentary: Statistical

Modeling for the Prediction of Infectious Disease Dissemination With

Special Reference to COVID-19

Spread.

Front. Public Health 9:783201.

doi: 10.3389/fpubh.2021.783201
Commentary: Statistical Modeling for the Prediction of Infectious Disease Dissemination With Special Reference to COVID-19 Spread

by Dhungana, H. N., and Ghimire, S. Front. Public Health. (2021) 9:735857. doi: 10.3389/fpubh.2021.735857

With reference to the quoted commentary (1) above, commentators observed, "In sections 'SI and SIS Models' and .... chance or probability." We would like to state that the commentators have misunderstood and misinterpreted the concept and definition of $\beta$. From the mentioned models, it may be seen that, $\beta$ is the per capita per unit time infection rate. It is a disease transmission coefficient or a transmission rate as described by Kirkeby et al. (2). Bailey (3), at page no. 20, and Bailey (4), at page no. 33, have defined $\beta$ as the infection rate. Chalub and Souza (5) have defined that, $\beta$ may be interpreted as a rate or as a probability among many other possible choices. Jagan et al. (6) have defined $\beta$ as the transmission rate; it is the number of infections per unit time per susceptible per infected. Further, Citron et al. (7) have defined as $\beta$ the transmission rate, published in "PNAS" and others (8-20).

Further, we have mentioned that $\beta$ estimates the spread rate, which shows the chance of transmission of the disease from an infectious individual to a susceptible one. Ucakan et al. (17) have explicitly expressed that the transmission coefficient, $\beta$ estimates the probability of getting the disease from an infectious individual to the susceptible and stated it as a probability that ranges from 0 to $1(0<\beta<1)$. On the other hand, Brauer and Castillo-Chavez (21) have defined $\beta$ as the product of the probability of the transmission per contact and the per capita contact rate. In addition, Chen (22) has also defined $\beta$ as the infection coefficient, the product of the average number of contacts within a given time period and the probability of infection for the contact between susceptible and infectious individuals. Thus, $\beta$ may take a value $>1$ and, therefore, in general, it may not be considered as a probability. Furthermore, one should be very careful about the choice of $\beta$ while defining diagrams for different epidemiological models. Moreover, Hethcote and Driessche (10) mentioned that the number of new cases per unit time shall be $\lambda S I / N$, which is called the standard incidence (23-27) with $\lambda$ as a contact rate. Another common incidence is the simple mass action incidence, $\beta S I$, where $\beta$ was defined as the transmission coefficient $(4,9)$. Additionally, Okabe and Shudo (16) have defined that $\beta S I$ represents the number of susceptible individuals that get infected per day. For more details, further references can be consulted $(28,29)$. 
In their next comment, they have wrongly pointed out "In section, "The Distribution Fitting," the .... biological interpretations." In the section "The Distribution Fitting" of our review on page no. 04 , we have clearly mentioned that "it is the growth rate of infection which determines the total number of infections which depends on the numerous factors (30)," and in the context of distribution fitting, we have mentioned that the infectious disease mainly depends on two factors, namely, the number of carriers and the time of infection as reported by Datta et al. (31). They have further mentioned, "In the section of "The Basic Reproduction Number"..... not the model." In the section "The Basic Reproduction Number," the commentators have wrongly stated the concept here. From the formula, it may be observed that, $R_{0}$ does not depend on time. Further, we have not mentioned $R_{0}$ as a rate anywhere in the review article. It is solely their imaginary creation. We have mentioned in the section that, $R_{0}$ is measured through the effective reproductive rate, denoted by $R$. Thus, we have mentioned, "effective reproductive number" $R$ as "effective reproductive rate" since it depends on time (32-38). Although for minimizing the ambiguity, the use of consistent terminology throughout the literature is required, and, therefore, we appreciate the commentators. Further, commentators have wrongly mentioned that we have stated $\xi$ as a model in the sub-section SIRS on page no. 9, however, $\xi$ is defined in subsection SEIRS on page no. 20. In our opinion, the sentence should be started with the word "In," which may be a typographical error, however, we have clearly mentioned that $\xi$ is the rate by which the recovered individuals become susceptible because of the loss of immunity, and $\xi$ is not a model. For more details, further references can be referred to $(15,39,40)$.

In their last comment, they have pointed out, "In the section, "Further Suggestions and Future Prospectives", ..... recommendations." Hajian-Tilaki (41) clearly observed that in designing epidemiologic studies, sample size calculation has an important role to detect an effect and achieve the desired precision in estimates of parameters of the interest (41-45). Therefore, it is a key factor that must be considered while

\section{REFERENCES}

1. Dhungana HN, Ghimire S. Commentary: Statistical modeling for the prediction of infectious disease dissemination with special reference to COVID-19 spread. Front Public Health. (2021) 9:735857. doi: 10.3389/fpubh.2021.73 5857

2. Kirkeby C, Halasa T, Gussmann M, Toft N, Græsbøll K. Methods for estimating disease transmission rates: evaluating the precision of Poisson regression and two novel methods. Sci Rep. (2017) 7:1-11. doi: 10.1038/s41598-017-09 209-x

3. Bailey NTJ. The Mathematical Theory of Epidemics. London: C. Griffin. RA652 B 3. p. 194. (1957).

4. Bailey NTJ. The Mathematical Theory of Infectious Diseases, 2nd Edn. New York, NY: Hafner (1975).

5. Chalub FACC, Souza MO. Discrete and continuous SIS epidemic models: a unifying approach. Ecol Complex. (2014) 18:83-95. doi: 10.1016/j.ecocom.2014.01.006 designing the study protocol (45). Small sample size will fail to provide a precise estimate and reliable answers to the policy makers (46). On the other hand, a large sample size than required will cause wastage of useful resources earmarked to the study (45). Malhorta and Indrayan (47) have recorded that, an adequate estimation of the correct required sample size is a must, especially in the case of such infectious diseases, for which the newly invented diagnostic tests are expensive to carry out. For any epidemiological study, the investigators must present the principles of sample size calculation to justify these numbers (44). Further, Hajian-Tilaki (48) also mentioned that, unfortunately, sample size calculations are rarely reported by clinical investigators for diagnostic studies $(49,50)$. The sample size calculation may be ignored wherever required, for instance, assumptions in household epidemic models for determining the transmissibility $\left(\mathrm{R}_{0}\right)$. This can sometimes be seen based on who infected whom however, it is only applicable to the infections with a long incubation period, such as AIDS and tuberculosis. Although, for the infections with a shorter incubation period, such as Influenza and COVID-19, we must meet the sample size conditions in order to estimate the growth rate. The commentators mentioned that in the section "Further Suggestions and Future Prospective," the compulsion of sample size determination can be avoided, however, we opined that, if certain researchers have not mentioned the assumptions regarding the sample size calculation, it does not mean that there is no need of it. Many a time, the determination of sample size is ignored, where strict ethical issues are not concerned, but it will harm in some sense or another, as mentioned by the more pragmatic scholars earlier $(45,46)$. Thus, it is highly recommended for an epidemiological study that the appropriate sample size calculation should be followed $(41,48,51-58)$.

\section{AUTHOR CONTRIBUTIONS}

SY and YA have together prepared the response to the commentary. Both the authors contributed to the article and approved the submitted version.

6. Jagan M. deJonge MS, Krylova O’Earn DJD. Fast estimation of time-varying infectious disease transmission rates. PLoS Comput Biol. (2020) 16:e1008124. doi: 10.1371/journal.pcbi.1008124

7. Citron DT, Guerra CA, Dolgert AJ, Wu SL, Henry JM, Sanchez CHM, et al. Comparing metapopulation dynamics of infectious diseases under different models of human movement. Proc Natl Acad Sci USA. (2021) 118:e2007488118. doi: 10.1073/pnas.2007488118

8. Anderson RM, Medley GP, May RM, Johnson AM. A preliminary study of the transmission dynamics of the human immunodefieiency virus, the causative agent of AIDS. IMA J Math Appl Med Biol. (1986) 3:229-63. doi: 10.1093/imammb/3.4.229

9. Anderson RM, May RM. Infectious Diseases of Humans: Dynamics and Control. Oxford: Oxford University Press (1991).

10. Hethcote HW, Driessche PVD. An SIS epidemic model with variable population size and a delay. J Math Biol. (1995) 34:177-94. doi: 10.1007/BF00178772

11. Liu M, Röst G, Vas G. SIS model on homogeneous networks with threshold type delayed contact reduction. Comput Math Appl. (2013) 66:1534-46. doi: 10.1016/j.camwa.2013.02.009 
12. Shausan, A. (2014) A Model for the Spread of an SIS Epidemic in a Human Population. A thesis submitted for the degree of Doctor of Philosophy, The University of Queensland.

13. Nakamura GM, Martinez AS. Hamiltonian dynamics of the SIS epidemic model with stochastic fluctuations. Sci Rep. (2019) 9:1584. doi: 10.1038/s41598-019-52351-x

14. Xuan W, Ren R, Pare PE, Ye M, Ruf S, Liu Ji. On a network SIS model with opinion dynamics. IFAC PapersOnLine. (2020) 53:2582-7. doi: $10.1016 /$ j.ifacol.2020.12.305

15. Giordano G, Blanchini F, Bruno R, Colaneri P, Filippo AD, Matteo $\mathrm{AD}$, et al. Modelling the COVID-19 epidemic and implementation of population-wide interventions in Italy. Nat Med. (2020) 26:855-60. doi: 10.1038/s41591-020-0883-7

16. Okabe Y, Shudo A. A mathematical model of epidemics-a tutorial for students. Mathematics. (2020) 8:1174. doi: 10.3390/math8071174

17. Ucakan Y, Gulen S, Koklu K. Analysing of tuberculosis in turkey through SIR, SEIR and BSEIR mathematical models. Math Comput Model Dyn Syst. (2021) 27:179-202. doi: 10.1080/13873954.2021.1881560

18. Nepomuceno EG, Peixoto MLC, Lacerda MJ, Campanharo ASLO, Takahashi RHC, Aguirre LA. Application of optimal control of infectious diseases in a model-free scenario. Sn Comput Sci. (2021) 2:405. doi: 10.1007/s42979-021-00794-3

19. Fayeldi T, Dinnullah RNI. Covid-19 sir model with nonlinear incidence rate. $J$ Phys Conf Ser. (2021) 1869:012113. doi: 10.1088/1742-6596/1869/1/012113

20. Cabrera M, Córdova-Lepe F, Gutiérrez-Jara JP, Vogt-Geisse K. An SIR-type epidemiological model that integrates social distancing as a dynamic law based on point prevalence and socio-behavioral factors. Sci Rep. (2021) 11:10170. doi: 10.1038/s41598-021-89492-x

21. Brauer F, and Castillo-Chavez, C. Basic models in epidemiology. In: Powell TM, Steele JH, editors. Ecological Time Series. Boston, MA: Springer (1994). p. 410-47. doi: 10.1007/978-1-4615-1769-6_19

22. Chen D. Modeling the spread of infectious diseases: a review. In: Chen D, Moulin B, Wu J, editors. Analyzing and Modeling Spatial and Temporal Dynamics of Infectious Diseases, 1st Edn. Hoboken, NJ: John Wiley \& Sons, Inc. (2014). p. 19-44.

23. Hethcote HW. Qualitative analysis of communicable disease models. Math Biosci. (1976) 28:335-56. doi: 10.1016/0025-5564(76)90132-2

24. Hethcote HW. A thousand and one epidemic models. In: Levin SA, ed. Frontiers in Mathematical Biology. New York: SpringerVerlag (1994). p. 50415. doi: 10.1007/978-3-642-50124-1_29

25. Hethcote HW, Van AJW. Epidemiological models with heterogeneous populations: proportionate mixing, parameter estimation and immunization programs. Math Biosci. (1987) 84:85-118. doi: 10.1016/0025-5564(87)90044-7

26. Mena-Lorca J, Hethcote HW. Dynamic models of infectious diseases as regulators of population sizes. J Math Biol. (1992) 30:693-716. doi: 10.1007/BF00173264

27. Gao LQ, Hethcote HW. Disease transmission models with density-dependent demographics, J. Math Biol. (1992) 30:717-31. doi: 10.1007/BF00173265

28. Wu JT, Leung K, Bushman M, Kishore N, Niehus R, de-Salazar PM, et al. Estimating clinical severity of COVID-19 from the transmission dynamics in Wuhan, China. Nat Med. (2020) 26:506-10. doi: 10.1038/s41591-020-0822-7

29. Zou L, Ruan F, Huang H, Hong Z, Yu J, Kang M, et al. SARS-CoV-2 viral load in upper respiratory specimens of infected patients. N Engl J Med. (2020) 382:970-1. doi: 10.1056/NEJMc2001737

30. Liang K. Mathematical model of infection kinetics and its analysis for COVID19, SARS and MERS infection, genetics and evolution. J Mole Epid Evolu Gen Infect Dis. (2020) 82:104306. doi: 10.1016/j.meegid.2020.104306

31. Datta R, Trivedi PK, Kumawat A, Kumar R, Bhradwaj I, Kumari N, et al. Statistical modeling of COVID-19 pandemic stages worldwide. Preprints. (2020) 2020:2020050319. doi: 10.20944/preprints202005.0319.v1

32. Caicedo-Ochoa Y, Rebellón-Sánchez DE, Peñaloza-Rallón M, Cortés-Motta HF, Méndez-Fandiño YR. Effective reproductive number estimation for initial stage of COVID-19 pandemic in Latin American Countries. Int J Infect Dis. (2020) 95:316-8. doi: 10.1016/j.ijid.2020.04.069

33. Inglesby TV. Public health measures and the reproduction number of SARSCoV-2. JAMA. (2020) 323:2186-7. doi: 10.1001/jama.2020.7878

34. Anderson R, Donnelly C, Hollingsworth D, Vegvari C, Baggaley R, Maddren R. Reproduction number $(\mathrm{R})$ and growth rate $(\mathrm{r})$ of the COVID-19 epidemic in the UK: methods of estimation, data sources, causes of heterogeneity, and use as a guide in policy formulation. The Royal Society. (2020). Available online at: https://royalsociety.org/-/media/policy/projects/set-c/set-covid-19-Restimates.pdf?la=en-GB\&hash=FDFFC11968E5D247D8FF641930680BD6

35. Rudiger S, Konigorski S, Rakowski A, Edelman JA, Zernick D, Thieme A, et al. Predicting the SARS-CoV-2 effective reproduction number using bulk contact data from mobile phones. Proc Natl Acad Sci USA. (2021) 118:e2026731118. doi: 10.1073/pnas.2026731118

36. Arroyo-Marioli F, Bullano F, Kucinskas S, Rondon-Moreno C. Tracking R of COVID-19: a new real-time estimation using the Kalman filter. PLoS ONE. 16:e0244474. doi: 10.1371/journal.pone.0244474

37. Lee Y, Lee DH, Know H-D, Kim C, Lee J. Estimation of the reproduction number of influenza A(H1N1)pdm09 in South Korea using heterogeneous models. BMC Infect. Dis. (2021) 21:658. doi: 10.1186/s12879-02106121-8

38. Thron C, Mbazumutima V, Tamayo LV, Todjihounde L. Cost effective reproduction number based strategies for reducing deaths from COVID-19. J. Math. Ind. (2021) 11:11. doi: 10.1186/s13362-021-00107-6

39. Kucharski A, Russell TW, Diamond C, Liu Y, Edmunds J, Funk S, et al. Early dynamics of transmission and control of COVID-19: a mathematical modelling study. Lancet Infect Dis. (2020) 20:553-8. doi: 10.1016/S1473-3099(20)30144-4

40. Khajanchi S, Bera S, Roy TK. Mathematical analysis of the global dynamics of a HTLV-I infection model, considering the role of cytotoxic T-lymphocytes. Math Comput Simul. (2021) 180:354-78. doi: 10.1016/j.matcom.2020.09.009

41. Hajian-Tilaki K. Sample size estimation in epidemiologic studies. Caspian J Intern Med. (2011) 2:289-98.

42. Detskey AS, Sackett DL. When was a negative clinical trial big enough? How many patients you needed depends on what you found. Arch Intern Med. (1985) 145:709-12. doi: 10.1001/archinte.1985.00360040141030

43. Houle TT, Penzien DB, Houle CK. Statistical power and sample size estimation for headache research: an overview and power calculation tools. Headache. (2005) 45:414-8. doi: 10.1111/j.1526-4610.2005.05092.x

44. Livingston EH, Cassidy L. Statistical power and estimation of the number of required subjects for a study based on the $t$-test. J Surg Res. (2005) 126:149-59. doi: 10.1016/j.jss.2004.12.013

45. Fitzner K, Heckinger E. Sample size calculation and power analysis: a quick review. Diabetes Educ. (2010) 36:701-7. doi: 10.1177/0145721710380791

46. Jones SR, Carley S, Harrison M. An introduction to power and sample size estimation. Emerg Med J. (2003) 20:453-8. doi: 10.1136/emj.20.5.453

47. Malhorta RK, Indrayan A. A simple nomogram for sample size for estimating sensitivity and specificity of medical tests. Indian J Ophthalmol. (2010) 58:51922. doi: 10.4103/0301-4738.71699

48. Hajian-Tilaki K. Sample size estimation in diagnostic test studies of biomedical informatics. J Biomed Inform. (2014) 48:193-204. doi: 10.1016/j.jbi.2014.02.013

49. Bachmann LM, Puhan MA. ter Riet G, Bossuyt PM. Sample size of studies on diagnostic accuracy: literature survey. BMJ. (2006) 332:1127-9. doi: 10.1136/bmj.38793.637789.2F

50. Bochmann F, Johmson Z, Azuara-Balanco A. Sample size in studies on diagnostic accuracy in ophthalmology: a literature survey. $\mathrm{Br} J$ Opthalmol. (2007) 91:898-9000. doi: 10.1136/bjo.2006.113290

51. Fosgate GT. Practical sample size calculations for surveillance and diagnostic investigations. J Vet Diagn Invest. (2009) 21:3-14. doi: $10.1177 / 104063870902100102$

52. Datta S, Funnell K, Ramuscak N. Improving the Sample Size Calculation Process for Peel Health: A Rapid Review of the Evidence. Region of Peel for You. (2012) Available online at: https://www.peelregion.ca/health/library/pdf/ Rapid-Review-Sample-Size-Report.pdf (accessed December 11, 2021).

53. Jalalian P. Sample Size Determination for Clinical Trials. (2014). Available online at: https://www.whitman.edu/Documents/Academics/Mathematics/ 2014/jalalipr.pdf (accessed December 11, 2021).

54. Herzog SA, Low N, Berghold A. Sample size considerations using mathematical models: an example with Chlamydia trachomatis infection and its sequelae pelvic inflammatory disease. BMC Infect Dis. (2015) 15:233. doi: 10.1186/s12879-015-0953-5

55. Blaizot S, Herzog SA, Abrams S, Theeten H, Litzroth A, Hens N. Sample size calculation for estimating key epidemiological parameters using serological 
data and mathematical modeling. BMC Med Res Methodol. (2019) 19:51. doi: 10.1186/s12874-019-0692-1

56. Wang X, Ji X. Sample size estimation in clinical research from randomized controlled trials to observational studies. Chest. (2020) 158:S12-20. doi: $10.1016 /$ j.chest.2020.03.010

57. Stevenson MA. Sample size estimation in veterinary epidemiologic research. Front Vet Sci. (2021) 7:539573. doi: 10.3389/fvets.2020.539573

58. Wohl S, Giles JR, Lessler J. Sample size calculation for phylogenetic case linkage. PLoS Comput Biol. 17:e1009182. doi: 10.1371/journal.pcbi.1009182

Conflict of Interest: The authors declare that the research was conducted in the absence of any commercial or financial relationships that could be construed as a potential conflict of interest.
Publisher's Note: All claims expressed in this article are solely those of the authors and do not necessarily represent those of their affiliated organizations, or those of the publisher, the editors and the reviewers. Any product that may be evaluated in this article, or claim that may be made by its manufacturer, is not guaranteed or endorsed by the publisher.

Copyright $\odot 2022$ Yadav and Akhter. This is an open-access article distributed under the terms of the Creative Commons Attribution License (CC BY). The use, distribution or reproduction in other forums is permitted, provided the original author(s) and the copyright owner(s) are credited and that the original publication in this journal is cited, in accordance with accepted academic practice. No use, distribution or reproduction is permitted which does not comply with these terms. 